\title{
O afeto sob a perspectiva do circumplexo: evidências de validade de construto
}

\author{
Ana Carla Crispim ${ }^{1}$ \\ University of Kent, Canterbury, Reino Unido \\ Roberto Moraes Cruz, Cassandra Melo Oliveira, Aline Battisti Archer \\ Universidade Federal de Santa Catarina, Florianópolis-SC, Brasil
}

\section{RESUMO}

O afeto é um estado de prazer ou desprazer com diferentes graus de ativação e é constantemente vivenciado pelas pessoas. Objetivou-se com este estudo buscar evidências de validade e precisão de um instrumento de medida para avaliação de afeto em uma amostra de atletas. A amostra foi composta de 330 atletas (87,6\% eram do sexo masculino), com média de idade de 21,8 anos ( $D P=5,36)$. O instrumento de medida utilizado na coleta de dados teve 26 pares de adjetivos que foram construídos com base em um circumplexo que representa um modelo possível para o afeto. A análise dos dados foi realizada por meio de procedimentos como estatística descritiva, análise fatorial e cálculo do alfa de Cronbach. Foram encontradas evidências de validade parciais para o instrumento de medida com três fatores. Sugere-se que sejam realizadas novas pesquisas para buscar evidências de validade e precisão acerca do afeto no contexto brasileiro.

Palavras-chave: afeto; psicometria; psicologia do esporte.

\section{ABSTRACT - Core affect and the circumplex approach: Evidence for construct validity}

Core affect is a feeling of pleasure or displeasure that varies in terms of activation, and it is constantly experienced by people. The aim of this study was to verify the validity and reliability evidence of a measurement instrument to assess core affect in a population of athletes. The sample was composed of 330 athletes $(87.6 \%$ are male), with a mean age of 21.8 years $(S D=5.36)$. The measurement instrument used for data collection included 26 pairs of adjectives based on a circumplex representing a possible model for core affect. Data analysis was performed using descriptive statistics, factor analysis, and Cronbach's alpha. Moreover, partial validity evidence was found for a 3-factor solution. In conclusion, further studies are recommended to search for evidence of validity and precision regarding core affect in the Brazilian population.

Keywords: core affect; psychometrics; sport psychology.

\section{RESUMEN - El afecto bajo la perspectiva del circumplejo: evidencia de la validez del constructo}

El afecto es un estado de placer o desplacer con grados variables de activación y se experimenta constantemente en la vida de las personas. El objetivo de este estudio fue buscar evidencia de la validez y exactitud de un instrumento de medida para evaluación de afecto en una muestra de atletas. La muestra fue de 330 atletas (87,6 \% varones) con una edad promedio de 21,8 años (DE=5,36). El instrumento de medición utilizado para la recolección de datos tuvo 26 pares de adjetivos que se basaban en un circumplejo que es un posible modelo de afecto. El análisis de datos se realizó mediante procedimientos tales como estadística descriptiva, análisis factorial y el cálculo de alfa de Cronbach. Se encontraron evidencias de validez parciales para el instrumento de medida con tres factores. Se sugiere que se realicen nuevas investigaciones para buscar evidencias de la validez y exactitud sobre el afecto en el contexto brasileño. Palabras clave: afecto; psicometría; psicología del deporte.

Referências mnemônicas, cognições e sentimentos são o resultado das experiências de vida das pessoas (Kahneman \& Riis, 2005). Quando um indivíduo, ao interagir com algum aspecto do ambiente, questionase "O que penso sobre ele?", "Será que gosto dele?" ou "Será que o odeio?", o componente afetivo sobre tal objeto é ativado (Kahneman, 2012). Comportamentos de lembrar-se de algo e agir estão intrinsicamente associados aos sentimentos das pessoas. Tais sentimentos podem ser caracterizados como afeto.

O afeto é compreendido como um estado de prazer ou descontentamento que possui algum grau de ativação e é vivenciado constantemente (Russell \& Barrett, 1999). É entendido, também, como um estado 
neurofisiológico conscientemente acessível, sendo uma combinação do hedônico, prazer ou desagrado, e da ativação, quietude ou vigor (Russell, 2003). Estados de prazer, de desgosto, de tensão, de calma, de energia e de cansaço são considerados exemplos de afeto (Ekkekakis, 2013).

Na década de 1960, Bradburn (1969) iniciou uma pesquisa sobre bem-estar partindo de duas dimensões: afeto positivo e afeto negativo. Essas dimensões tornaram-se, posteriormente, a base de pesquisas acerca da mensuração do afeto, resultando na construção, por Watson e Tellegen (1988), da Positive and Negative Affect Schedule (PANAS). A mudança da expressão e concepção de bem-estar para afeto ficou evidente na década de 1980, quando ocorreram avanços conceituais e metodológicos sobre o afeto (Russell, 1980; Watson, Clark, \& Tellegen, 1988). Ainda que a contribuição teórica da PANAS seja semelhante à de Bradburn (1969), Watson e Tellegen (1988) construíram a PANAS considerando o humor como seu construto principal de exame.

Apesar de o teste PANAS possuir evidências de validade, não é possível afirmar que ele mensure o afeto em relação a todas as suas dimensões, conforme foi constatado posteriormente pelos próprios autores (Ekkekakis, 2013; Watson, Wiese, Vaidya, \& Tellegen, 1999). Quando o conteúdo da PANAS é avaliado, apenas afetos com alta e moderada ativações são medidos pelos itens, como pode ser visto nas duas escalas PANAS traduzidas no Brasil (Carvalho et al., 2013; Zanon \& Hutz, 2014). Tal característica não tem relação apenas com as propriedades do objeto de mensuração da escala, mas também pode vir a afetar futuras associações entre diferentes construtos, quando escores derivados da PANAS são utilizados. Além disso, a estrutura fatorial da PANAS ainda levanta dúvidas no tocante à ortogonalidade dos seus fatores e à validade dos seus itens (Ekkekakis, 2013; SeibPfeifer, Pugnaghi, Beauducel, \& Leue, 2017). Dessa forma, inferências com base em escores dessa escala devem levar em conta as limitações citadas.

Na década de 1980, Russell (1980) apresentou outra contribuição teórica para a compreensão do afeto, por meio da caracterização de duas dimensões: valência e ativação. As combinações dessas duas dimensões, em diferentes graus, teriam como resultado as experiências afetivas. O modelo teórico resultante, denominado de circumplexo de Russell (1980), teve continuidade em estudos posteriores (Carroll, Yik, Russell, \& Barrett, 1999; Russell \& Barrett, 1999; Russell, 2003; Yik, Russell, \& Steiger, 2011). Estudos como os de Carroll et al. (1999) e Yik et al. (2011) trouxeram avanços com relação a como modelar as variáveis de afeto em um circumplexo e como sentimentos são entendidos por esse modelo; pesquisas como a de Russell e Barrett (1999) visavam indicar novas perspectivas sobre como medir afeto e discutir esse construto teoricamente. Ao longo das décadas houve mudanças importantes na teoria do afeto, especialmente ao se reconhecer que os estudos sobre humor pertenciam ao campo conceitual do afeto.

O afeto, para Russell (1980), é compreendido por meio do circumplexo (Figura 1). Suas dimensões são bipolares e ortogonais, sendo nomeadas de valência (prazer ou desprazer) e ativação percebida (alta ou baixa). O circumplexo é uma estrutura ordenada em que todos os testes apresentam um mesmo nível de complexidade e diferem em termos do tipo de habilidade que eles medem. Quando um construto pode ser representado por um circumplexo, sua matriz de correlações apresenta um padrão de correlações fortes perto da diagonal e, conforme as correlações se afastam da diagonal, elas ficam mais fracas, até que voltam a ficar fortes. Esse padrão de correlações repete-se em toda a matriz, e, por isso, pontos próximos no circumplexo são correlacionados fortemente (Guttman, 1954).

A dimensão valência está relacionada à codificação do ambiente como prazeroso ou desprazeroso. Para o estímulo em um determinado momento, o sujeito pode atribuir um significado: bom ou ruim; útil ou prejudicial; recompensador ou ameaçador (Barrett, 2006). A ativação, por sua vez, é a dimensão da experiência que corresponde à mobilização ou energia dispensada; ou seja, é representada por um continuum, desde a baixa ativação, representada por sono, até a ativação alta, representada pela excitação (Russell \& Barrett, 1999).

Estados afetivos que são próximos no circumplexo representam uma combinação similar de valência e ativação percebida; já estados afetivos posicionados diametricamente longe um do outro diferem em termos de valência e ativação (Russell, 1980). Assim, as quatro variáveis alocadas diagonalmente não são dimensões, mas ajudam a definir os quadrantes no espaço do circumplexo (Russell, 1980).

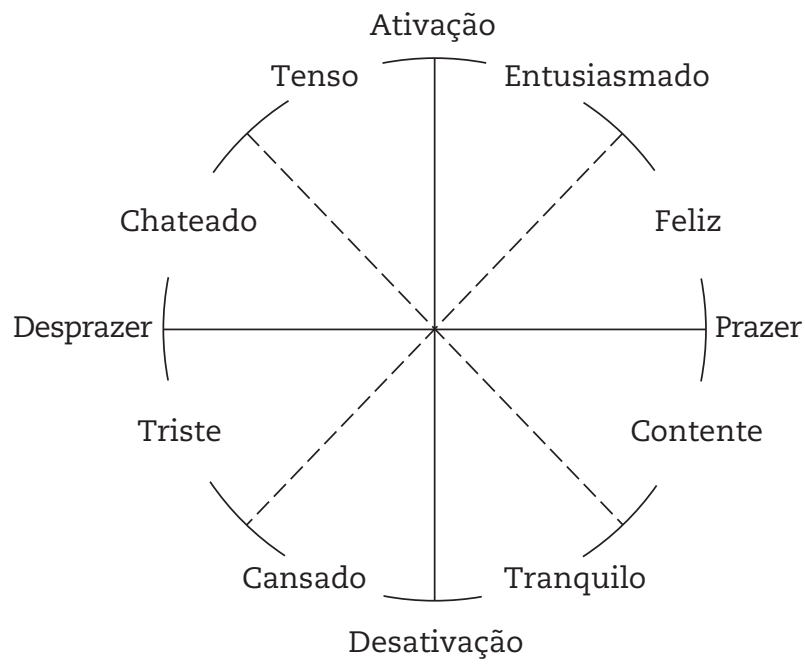

Nota: Traduzido do inglês e adaptado de Carroll et al. (1999).

Figura 1. Dimensões e variáveis constituintes do circumplexo de Russell 
Ao longo dos anos, diversos instrumentos foram criados (Betella \& Verschure, 2016; Kammann \& Flett, 1983; Mehrabian \& Russell, 1974; Quirin, Kazén, \& Kuhl, 2009; Scherer, Shuman, Fontaine, \& Soriano, 2013; Watson et al., 1988; Yik et al., 2011) com base nas proposições de Russell (1980) e Bradburn (1969). Entre eles, os instrumentos de Kammann e Flett (1983), Watson et al. (1988) e Quirin et al. (2009) possuem estruturas teóricas semelhantes e baseadas nas dimensões afetos positivos e afetos negativos, enquanto os instrumentos de Mehrabian e Russell (1974), Betella e Verschure (2016), Scherer et al. (2013) e Yik et al. (2011) apresentam fundamentação teórica similar à do circumplexo, com base nas dimensões valência e ativação. De todas as escalas identificadas na literatura especializada, apenas a PANAS (Carvalho et al., 2013; Zanon \& Hutz, 2014) possui evidências de validade no Brasil.

Ao testar estatisticamente as dimensões valência, ativação, afeto positivo e afeto negativo em um mesmo modelo, foi identificado que, por mais que tais dimensões demonstrem diferenças conceituais, enfaticamente elas apresentam aspectos complementares quanto ao construto afeto e não distintos entre si. A análise do espaço bidimensional com modelos que estabeleciam dimensões relacionadas a valência/ativação ou a afeto positivo/afeto negativo demonstrou que todas as dimensões poderiam ser representadas simultaneamente em um espaço com duas dimensões. Dessa forma, elas estavam próximas entre si no circumplexo (Yik, Russell, \& Barrett, 1999).

Com base nos modelos teóricos analisados, as combinações das dimensões geraram seis categorias: afeto positivo com ativação alta (eufórico, entusiasmado); afeto positivo com ativação moderada (gratificante, satisfeito); afeto positivo com ativação baixa (sereno, calmo); afeto negativo com ativação alta (chateado, em sofrimento); afeto negativo com ativação moderada (miserável, descontente); e afeto negativo com ativação baixa (letárgico, depressivo) (Carroll et al., 1999). Essas dimensões foram posteriormente adaptadas por Yik et al. (2011) em um novo modelo, o 12-PAC (12-Point Affect Circumplex). Nesse modelo, o afeto pode ser mensurado por meio de 12 pontos concernentes às combinações entre valência e ativação.

Além de evidências de validade de construto, evidências de validade com construtos externos ao afeto também foram fornecidas no estudo do modelo 12-PAC (Yik et al., 2011). A dimensão extroversão do modelo dos Cinco Grandes Fatores (CGF) correlacionou-se $(r=0,35)$ com a posição $35^{\circ}$ (alta ativação e afeto positivo), enquanto a dimensão neuroticismo se correlacionou $(r=0,48)$ com a posição $182^{\circ}$ (ativação neutra e afeto negativo). Além do CGF, o construto ansiedade-traço correlacionou-se $(r=0,60)$ com a $179^{a}$ posição (ativação neutra e afeto negativo), e a escore do construto depressão (Inventário de Depressão de Beck) correlacionou-se $(r=0,58)$ com a $182^{\mathrm{a}}$ posição.

Com base nos resultados encontrados na literatura, foi selecionado o modelo de Carroll et al. (1999) para construir um novo instrumento de medida sobre afeto no
Brasil. A escolha desse modelo deu-se pela necessidade de desenvolver um instrumento de medida sobre afeto que considerasse o construto em diferentes níveis de ativação para ser utilizado, inicialmente, no contexto do esporte. Como destacado anteriormente, a escala PANAS possui deficiências com relação à dimensão ativação, e, por isso, foi necessária a construção de um novo instrumento de medida. Dado que o desenvolvimento do novo instrumento era parte de um projeto sobre avaliação de afetos no contexto do esporte, uma amostra de atletas foi considerada como mais apropriada para o estudo, no entanto destaca-se que os itens podem constituir parte do processo de medida do afeto em contextos diferentes do esportivo.

Dessa forma, o objetivo deste estudo foi buscar evidências de validade e precisão de um instrumento de medida para avaliação de afeto em uma amostra de atletas.

\section{Método}

\section{Participantes}

A amostra por conveniência foi composta por 330 atletas, em sua maioria do sexo masculino $(87,6 \%)$, com média de idade de 21,8 anos $(D P=5,36)$. O nível de escolaridade dos participantes foi ensino médio completo (56,8\%), ensino fundamental (20,7\%) e ensino superior (19,8\%). A maioria mora na Região Sul do país $(87,4 \%)$, e estão distribuídos por 56 cidades como local de moradia. Foram identificadas 13 modalidades de esporte entre os participantes: artes marciais $(3 \%)$, atletismo $(6,1 \%)$, ciclismo $(0,3 \%)$, fisiculturismo $(0,6 \%)$, futebol $(30,6 \%)$, futebol americano $(20,9 \%)$, futsal $(1,5 \%)$, handebol $(3,6 \%)$, motocross $(0,3 \%)$, natação $(0,3 \%)$, tênis $(9,1 \%)$, triatlo $(0,9 \%)$ e vôlei $(22,7 \%)$. Futebol, futebol americano e vôlei foram as modalidades com maior quantidade de participantes $(74,2 \%)$. O tempo de prática esportiva variou entre 2 meses e 27 anos, com média de 8,7 anos $(D P=5,2)$ de prática na modalidade citada.

\section{Instrumentos}

Instrumento de medida sobre afeto. Para construção do instrumento de medida, foi realizada primeiramente uma revisão de literatura nacional e internacional acerca do construto afeto. A perspectiva teórica escolhida foi a do circumplexo do afeto (Russell, 2003), na qual é proposta a utilização de duas dimensões valência e ativação - para a explicação do construto. As combinações das duas dimensões do afeto formam categorias com diferentes níveis de ativação e de valência, como proposto por Carroll et al. (1999). Com essa lógica, o instrumento foi construído formando três categorias:

1. afeto negativo com ativação baixa versus afeto positivo com ativação alta;

2. afeto negativo com ativação alta versus afeto positivo com ativação baixa;

3. afeto negativo com ativação moderada versus afeto positivo com ativação moderada. 
Os itens foram construídos com o formato de diferencial semântico (escala de 7 pontos). $\mathrm{O}$ formato de diferencial semântico possui duas âncoras verbais que representam polos extremos de um continuum. Como esse formato, possibilita-se que categorias opostas de um continuum sejam utilizadas em uma mesma escala, e esse formato foi avaliado como coerente à categorização dos itens (combinações de valência e ativação). Inicialmente foram construídos 24 itens para o instrumento de medida. A versão final do instrumento foi reajustada com base nos resultados do estudo piloto para a busca de evidências de validade semântica e foi composta de 26 itens (Crispim, Roettgers, \& Cruz, 2014).

\section{Procedimentos}

Após a aprovação do Comitê de Ética da Universidade Federal de Santa Catarina (UFSC), parecer $n^{\circ} 711.498$, as equipes técnicas dos atletas foram contatadas para que a pesquisa pudesse ter início. Para que os atletas participassem da investigação, foi necessário que assinassem o Termo de Consentimento Livre e Esclarecido (TCLE). As aplicações ocorreram nos ambientes escolhidos pelas equipes técnicas dos atletas, em horários que não interferissem na rotina de treinos ou competições.

\section{Tratamento e análise de dados}

Os dados foram tratados e analisados com base no pacote estatístico Mplus versão 7.1. Para busca de evidências de validade de estrutura interna do construto, os itens do instrumento de medida foram submetidos à análise fatorial exploratória, utilizando o método Weighted Least Squares Means and Variance Adjusted (WLSMV), com rotação oblíqua geomin para dados ordinais. Como o procedimento padrão de análise fatorial identifica os dados como intervalares, a análise fatorial foi realizada respeitando as características originais dos dados, ou seja, suas características ordinais. A rotação oblíqua geomin foi escolhida uma vez que permite que os fatores se correlacionem (McDonald, 2014). Para confirmação do número de fatores a serem retidos, foram feitos os seguintes procedimentos: o screeplot; o Root Mean Square Error of Approximation (RMSEA), com ponto de corte menor ou igual a 0,05 ; o Comparative Fit Index (CFI), com ponto de corte maior ou igual a 0,90; e o Standardized Root Mean Square Residual (SRMR), menor ou igual a 0,05 (Byrne, 2012; Kline, 2016). O ponto de corte do erro padrão para as cargas fatoriais foi calculado por meio da seguinte Equação 1, como sugere (McDonald, 2014):

$$
m=\frac{1}{\sqrt{N}}
$$

Nessa equação, $m$ representa o ponto de corte padrão, e $N$ refere-se à amostra total. A precisão do instrumento de medida foi verificada por intermédio do coeficiente de Cronbach. O erro padrão da medida (EPm) para os escores foi calculado pela Equação 2 (McDonald, 2014):

$\operatorname{EPm}(\gamma)=D P(Y) \sqrt{1}-\alpha$

$\mathrm{Na}$ fórmula, $D P(Y)$ refere-se ao desvio padrão da medida e $\alpha$ ao valor do alfa de Cronbach.

\section{Resultados}

Foram testados modelos para dois, três e quatro fatores com dados categóricos e ordinais, uma vez que a escala de pontos dos itens possuía propriedades ordinais. Para verificar qual modelo se adequava melhor aos dados, foram realizados o screeplot, o RMSEA, o CFI e o SRMR. $\mathrm{O} \chi^{2}$ foi verificado, no entanto não foi considerado como fator de decisão na análise, por conta da influência do tamanho da amostra em seu valor (Tabela 1).

O modelo de dois fatores apresentou valores aceitáveis de RMSEA e CFI, mas o SRMR apresentou média de resíduos acima de 0,05 . Os modelos de três e quatro fatores apresentaram valores aceitáveis de RMSEA, CFI e SRMR. De acordo com o screeplot, existe uma quebra (ponto de inflexão) no terceiro ponto - sugerindo a extração de dois fatores - e um ponto de inflexão no quarto ponto, o que sugere a extração de três fatores. Como a extração de dois fatores apresentou o SRMR de 0,06 , a extração de três e de quatro fatores foi considerada a mais adequada, tanto por motivos estatísticos quanto por motivos teóricos.

De forma complementar, foram analisados os resíduos das correlações, as cargas fatoriais (Tabela 2) e o erro padrão das cargas fatoriais para dois, três e quatro fatores. Como o valor de corte do SRMR é 0,05, o mesmo ponto de corte foi aplicado para os resíduos da matriz de correlações. Foram encontrados resíduos acima de 0,05 para dois, três e quatro fatores, com menor frequência nas matrizes de três e quatro fatores, levantando a hipótese de que, como alguns itens têm adjetivos com conotação similar, parte da variância dos itens não é explicada pelos fatores, mas sim pela relação entre o conteúdo dos próprios itens.

Tabela 1

Distribuição dos valores de adequação nos modelos de dois, três e quatro fatores

\begin{tabular}{llcccc}
\hline \multirow{2}{*}{$\begin{array}{l}\text { Número } \\
\text { de fatore }\end{array}$} & \multicolumn{2}{c}{$\chi^{2}$} & & & \\
& $\chi^{2}(\mathrm{df})$ & $p$ & & & \\
& RMSEA & CFI & SRMR \\
3 & $849,366(274)$ & 0,0001 & 0,080 & 0,920 & 0,062 \\
4 & $656,999(250)$ & 0,0001 & 0,070 & 0,944 & 0,049 \\
4 & $508,548^{*}(227)$ & 0,0001 & 0,061 & 0,961 & 0,041 \\
\hline
\end{tabular}

Nota: RMSEA=Root Mean Square Error of Approximation; $\mathrm{CFI}=$ Comparative Fit Index; SRMR=Root Mean Square Residual. 
Por fim, analisou-se a magnitude $(m)$ do erro padrão das cargas fatoriais. O valor limítrofe foi 0,06 para a amostra de 330 participantes. Itens que apresentam valores acima de 0,06 podem ser um indício de que o modelo proposto para os dados não é identificado (McDonald, 2014). Dessa forma, a análise dos erros padrão é essencial para identificar se o modelo proposto está de acordo com os dados encontrados e se existem itens problemáticos com relação ao seu valor de erro padrão (McDonald, 2014). A solução de dois fatores não apresentou valores acima de 0,06 , enquanto as soluções de três e quatro fatores apontaram erros padrão acima de 0,06 . A solução de quatro fatores indicou valores de adequação menores, mas essa solução possui maior quantidade de erros padrão nas cargas fatoriais acima do valor de corte, sendo um problema para a estrutura fatorial. $\mathrm{Na}$ solução de três fatores, os valores dos erros padrão ultrapassaram o valor de corte nos itens 14 ("insatisfeito/satisfeito") e 25 ("triste/feliz"). Com base nisso, foi decidido realizar a interpretação dos fatores e das cargas fatoriais por meio das três dimensões, sendo esse o modelo mais parcimonioso.

Teoricamente, foi proposto que os itens 1 a 10 se referiam ao fator 1 (afetos prazerosos com ativação alta), os itens 11 a 19 ao fator 2 (afetos prazerosos com ativação baixa) e os itens 20 a 26 ao fator 3 (afetos prazerosos com ativação moderada). Como o afeto é conhecido por ter a estrutura de circumplexo, espera-se que alguns itens apresentem cross-loadings, ou seja, cargas fatoriais significativas em mais de um

Tabela 2

Distribuição das cargas fatoriais para dois, três e quatro fatores

\begin{tabular}{|c|c|c|c|c|c|c|c|c|c|}
\hline \multirow{2}{*}{ Itens } & \multicolumn{2}{|c|}{ Dois fatores } & \multicolumn{3}{|c|}{ Três fatores } & \multicolumn{4}{|c|}{ Quatro fatores } \\
\hline & 1 & 2 & 1 & 2 & 3 & 1 & 2 & 3 & 4 \\
\hline 1 Inativo/ativo & 0,66 & $-0,12$ & 0,66 & $-0,09$ & 0,02 & 0,74 & $-0,02$ & $-0,03$ & 0,07 \\
\hline 2 Desprazeroso/prazeroso & 0,67 & $-0,02$ & 0,62 & 0 & 0,08 & 0,38 & 0,29 & 0 & 0,11 \\
\hline 3 Cansado/ativo & 0,72 & $-0,07$ & 0,71 & $-0,04$ & 0,02 & 0,73 & 0,07 & 0,02 & 0,06 \\
\hline 4 Abatido/eufórico & 0,60 & $-0,2$ & 0,60 & $-0,18$ & 0,02 & 0,52 & 0,13 & $-0,15$ & 0,05 \\
\hline 5 Preguiçoso/enérgico & 0,74 & $-0,12$ & 0,94 & $-0,01$ & $-0,27$ & 0,63 & 0,4 & 0,01 & $-0,23$ \\
\hline 6 Desinteressado/interessado & 0,72 & $-0,01$ & 0,78 & 0,05 & $-0,08$ & 0,09 & 0,73 & $-0,01$ & $-0,04$ \\
\hline 7 Indiferente/entusiasmado & 0,76 & $-0,07$ & 0,75 & $-0,04$ & 0,02 & 0,06 & 0,72 & $-0,11$ & 0,06 \\
\hline 8 Desmotivado/motivado & 0,81 & 0,03 & 0,82 & 0,08 & $-0,01$ & $-0,01$ & 0,89 & $-0,02$ & 0,03 \\
\hline 9 Depressivo/animado & 0,74 & 0,08 & 0,79 & 0,15 & $-0,06$ & 0,02 & 0,82 & 0,06 & $-0,02$ \\
\hline 10 Desesperançoso/esperançoso & 0,62 & 0,05 & 0,61 & 0,08 & 0,01 & 0,10 & 0,57 & 0,03 & 0,04 \\
\hline 11 Tenso/relaxado & $-0,26$ & 0,79 & $-0,18$ & 0,83 & $-0,17$ & $-0,01$ & $-0,13$ & 0,81 & $-0,14$ \\
\hline 12 Nervoso/calmo & $-0,17$ & 0,81 & 0,03 & 0,90 & $-0,35$ & 0,13 & $-0,03$ & 0,89 & $-0,31$ \\
\hline 13 Irritado/sereno & 0,04 & 0,62 & $-0,02$ & 0,62 & 0,04 & 0,09 & $-0,06$ & 0,61 & 0,07 \\
\hline 14 Insatisfeito/satisfeito & 0,50 & 0,25 & 0,17 & 0,15 & 0,46 & 0,07 & 0,14 & 0,14 & 0,46 \\
\hline 15 Impaciente/paciente & 0 & 0,70 & $-0,10$ & 0,69 & 0,07 & $-0,02$ & $-0,04$ & 0,68 & 0,09 \\
\hline 16 Mal-humorado/bem-humorado & 0,30 & 0,39 & 0 & 0,30 & 0,38 & 0,10 & $-0,07$ & 0,31 & 0,39 \\
\hline 17 Pressionado/descansado & 0,13 & 0,62 & 0,08 & 0,63 & 0,02 & 0,01 & 0,13 & 0,61 & 0,04 \\
\hline 18 Inquieto/quieto & $-0,16$ & 0,51 & 0 & 0,58 & $-0,28$ & $-0,05$ & 0,10 & 0,56 & $-0,27$ \\
\hline 19 Chateado/contente & 0,64 & 0,19 & 0,08 & $-0,01$ & 0,79 & $-0,03$ & 0,13 & $-0,03$ & 0,79 \\
\hline 20 Agoniado/sossegado & 0 & 0,72 & $-0,03$ & 0,74 & $-0,01$ & $-0,1$ & 012 & 0,70 & 0 \\
\hline 21 Aborrecido/alegre & 0,57 & 0,29 & 0,03 & 0,12 & 0,74 & 0,07 & 0 & 0,12 & 0,74 \\
\hline 22 Indisposto/disposto & 0,74 & 0,12 & 0,60 & 0,10 & 0,21 & 0,33 & 0,34 & 0,09 & 0,24 \\
\hline 23 Inseguro/seguro & 0,37 & 0,49 & 0,34 & 0,51 & 0,02 & 0,10 & 0,31 & 0,47 & 0,04 \\
\hline 24 Angustiado/aliviado & 0,24 & 0,53 & 0,15 & 0,53 & 0,09 & $-0,11$ & 0,31 & 0,48 & 0,10 \\
\hline 25 Triste/feliz & 0,66 & 0,17 & 0,19 & $-0,01$ & 0,67 & 0,17 & 0,06 & $-0,01$ & 0,67 \\
\hline 26 Aflito/tranquilo & 0,11 & 0,71 & $-0,02$ & 0,69 & 0,13 & $-0,09$ & 0,13 & 0,66 & 0,14 \\
\hline
\end{tabular}


fator. Apesar de não existir um ponto de corte claro com relação aos valores das cargas fatoriais, neste estudo o ponto de corte estabelecido foi 0,32 ou superior, uma vez que isso indica que $10 \%$ ou mais da variância do item foi explicada pelo fator (Tabachnick \& Fidell, 2013). O fator 1 carregou 11 itens, com cargas fatoriais entre 0,60 e 0,94, sendo esse fator referente a afetos prazerosos com alta ativação. $\mathrm{O}$ item que melhor representou esse fator, ou seja, o item-âncora, foi o item 5 ("preguiçoso/enérgico"). Apesar de o item 23 ("inseguro/seguro") ter carregado no fator 1 $(\lambda=0,34)$, ele apresentou maior cross-loading com o fator $2(\lambda=0,51)$ e, por isso, não foi mantido no fator 1 . $O$ fator 2 refere-se a afetos prazerosos com baixa ativação e carregou 10 itens com cargas fatoriais entre 0,51 e 0,9 , sendo o item 12 ("nervoso/calmo") considerado o item-âncora. O fator 3 carregou cinco itens, com cargas fatoriais entre 0,37 e 0,79 , e seu item-âncora foi o item 19 ("chateado/contente"). Os cinco itens $(14,16,19,21$ e 25) carregados no fator 3 pertencem à categoria 3 (Carroll et al., 1999), a qual contém afetos de ativação moderada.

Ao longo da análise fatorial com dois, três e quatro fatores, foi identificado que alguns itens estavam em categorias diferentes das propostas teoricamente. $\mathrm{O}$ item 22 ("indisposto/disposto") carregou no fator 1, o que trouxe evidências de que o afeto "disposto" teve conotação de uma mobilização de energia maior do que a moderada. O fator 2 carregou quatro novos itens ("agoniado/sossegado", "inseguro/seguro", "angustiado/aliviado" e "aflito/ tranquilo"), que foram propostos como de ativação moderada. Pesquisa realizada por Yik et al. (2011) mostrou que afetos prazerosos desses pares de adjetivos foram também referidos como de baixa ativação.

O fator 3 foi o fator mais afetado estruturalmente, uma vez que a maior parte dos itens com ativação moderada carregou no fator 1 e 2 . O item 14 ("insatisfeito/satisfeito") e o item 16 ("mal-humorado/bem-humorado"), antes da categoria 2, carregaram predominantemente no fator 3, o que indicou que a ativação desses itens é moderada, como visto em outros estudos (Yik et al., 2011). Como dois itens desse fator obtiveram valores acima do esperado nos erros padrão nas cargas fatoriais, sugere-se que futuras interpretações acerca desse fator sejam realizadas de forma cautelosa.

As correlações obtidas pelos fatores 1 e $3(r=0,62)$, 2 e $3(r=0,39)$ e 1 e $2(r=0,24)$ mostraram compartilhamento de variância entre os fatores, sugerindo que eles medem um construto em comum. Na matriz de correlação de todos os itens, essa relação foi identificada por meio das correlações com valores entre $r=0,20$ e $r=0,40$ entre os itens do fatores 1 e 3 . Como afetos próximos no circumplexo são similares em termos de valência e ativação (Carroll et al., 1999), foi hipotetizado que parte dos afetos dos fatores 1 e 3 está próxima no circumplexo e que, por isso, apresentou correlação forte.
Para finalizar a análise, foi calculada a fidedignidade das dimensões encontradas na análise fatorial. A qualidade da fidedignidade foi identificada por meio do alfa de Cronbach, técnica que verifica a consistência interna de um teste mediante a análise da consistência interna dos itens (Pasquali, 2013). O cálculo foi realizado com os itens dos fatores previstos pela análise fatorial exploratória, uma vez que uma das premissas para utilização do alfa de Cronbach é que o cálculo deve ser feito com itens que se refiram a um mesmo atributo (McDonald, 2014).

No geral, os coeficientes de Cronbach das categorias foram considerados consistentes $(\alpha=0,76$ a 0,88 ), como é observado na Tabela 3 . O valor de alfa do item 18 indicou que, caso esse item fosse retirado, o alfa total do fator 2 não mudaria; da mesma forma, se o item 16 fosse retirado, o alfa do fator 3 aumentaria para 0,79 . O item 16 apresentou a menor carga fatorial para o fator 3, o que é um indicativo de que esse item pode ter problemas com relação ao seu conteúdo. Outra hipótese se refere ao fato de que tanto o item 16 quanto o item 18 podem estar medindo outro construto, como o humor, o que pode dar margem para erros em futuras inferências, caso eles sejam mantidos na escala (Ekkekakis, 2013). No presente estudo, os itens foram mantidos nas escalas, pois não existem evidências suficientes para excluí-los.

Com os itens definidos em cada fator, escores foram criados por meio das médias de pontuação dos itens em cada fator (Pasquali, 2013). O fator 1 foi identificado como similar à categoria 1 proposta inicialmente e refletiu a bipolaridade entre os afetos desprazerosos com baixa ativação (depressão, letargia e cabisbaixo) com afetos prazerosos e alta ativação (entusiasmado, enérgico e eufórico). O escore médio foi 6,1 pontos $(D P=0,8)$. O fator 2 correspondeu aos itens originalmente propostos para a categoria 2 do instrumento e identificou afetos desprazerosos de alta ativação (nervosismo, tensão e irritação) com afetos prazerosos de baixa ativação (calma, serenidade e relaxado). O escore médio desse fator foi 4,7 pontos $(D P=1,1)$. O fator 3 representou afetos agradáveis e desagradáveis com ativação moderada (insatisfeito/satisfeito; chateado/contente; aborrecido/alegre; triste/feliz). O escore médio desse fator foi de 5,9 pontos $(D P=0,9)$.

Tabela 3

Distribuição do alfa de Cronbach por fator para amostra completa

\begin{tabular}{lccc}
\hline Fatores & $\begin{array}{c}\text { Número } \\
\text { de itens }\end{array}$ & $\begin{array}{c}\text { Alfa de } \\
\text { Cronbach }\end{array}$ & $\begin{array}{c}\text { Erro padrão } \\
\text { da medida }\end{array}$ \\
\hline Fator 1 & 11 & 0,88 & 0,26 \\
Fator 2 & 10 & 0,86 & 0,41 \\
Fator 3 & 5 & 0,76 & 0,43 \\
\hline
\end{tabular}




\section{Discussão}

A estrutura do circumplexo foi testada por meio da análise fatorial exploratória, de forma a estudar o construto afeto como uma estrutura com três categorias. Mudanças com relação à posição dos itens foram revistas com base na teoria proposta e em resultados de outros estudos, como o de Yik et al. (2011). A coerência com outros estudos sobre o circumplexo é uma parte importante na procura por evidências de validade, uma vez que o modelo do circumplexo de Russell (1980) não havia sido aplicado no Brasil.

Apenas evidências parciais de validade de construto foram encontradas neste estudo, no entanto destaca-se que a presente pesquisa teve caráter estritamente exploratório, já que parte dos itens utilizados não havia sido testada em populações brasileiras anteriormente. Assim, a estrutura de três fatores foi tida como a solução mais parcimoniosa para os dados encontrados, o que reforça a ideia de que os adjetivos, os quais representam afetos, são influenciados pelas dimensões valência e ativação.

Teoricamente, o afeto é um construto complexo (Russell, 1980), e, quando itens de instrumentos de medida de construtos complexos são analisados, é possível ver que os itens são influenciados em algum grau por uma ou mais de suas dimensões constitutivas (McDonald, 2014). Empiricamente, as evidências mostram indícios na mesma direção, pois cross-loadings foram identificadas na matriz de cargas fatoriais. Deve-se levar em consideração que os adjetivos foram propostos para diferir em termos de ativação nesse instrumento, o que pode ter influenciado na força das cross-loadings, já que cada fator representa categorias diferentes de ativação e valência.

$\mathrm{O}$ uso da teoria do circumplexo do afeto segue uma perspectiva diferente daquelas encontradas nas pesquisas brasileiras, que tendem majoritariamente a utilizar os conceitos de afetos positivos e negativos e a escala PANAS (Carvalho et al., 2013; Zanon \& Hutz, 2014). Entende-se que construir novas possibilidades teóricas e instrumentais é uma condição que permite, a médio e longo prazos, o desenvolvimento e o aperfeiçoamento da medida do afeto para a população em geral, e não só para atletas.

Apesar de o instrumento ter sido aplicado em uma população de atletas e sua instrução incluir o estímulo de competições, ele não necessariamente precisa continuar sendo testado apenas nessa população. De fato, ao verificar as médias de escores da amostra de atletas, percebe-se que elas não diferem fortemente da população geral. Por exemplo, os atletas reportaram notoriamente mais sentimentos positivos do que negativos, o que é uma tendência que pode ser observada em outros estudos sobre afeto que foram realizados na população geral brasileira (Segabinazi et al., 2012; Zanon, Bastianello, Pacico, \& Hutz, 2013). Tal evidência não demonstra que os resultados desse estudo podem ser generalizados para toda a população, mas futuros estudos com outras amostras podem considerar, como ponto de partida, as evidências de validade de construto descobertas neste estudo.

Destaca-se que limitações como o não balanceamento dos grupos em termos de gênero e o caráter exploratório da análise impõem restrições nas inferências do presente estudo. O caráter exploratório da análise faz com que a derivação de escores fatoriais não seja possível, antes que um modelo confirmatório seja testado nos dados. Por consequência, buscar evidências de validade com outros construtos tornou-se inviável, uma vez que escores brutos ou escores baseados em médias contêm erro. Além disso, a predominância do sexo masculino na amostra (amostra por conveniência) inviabiliza a comparação de escores de outras variáveis com quaisquer outros grupos que não sejam atletas do sexo masculino.

Novos estudos podem ser realizados com o objetivo de buscar evidências de validade na população geral brasileira, de forma que grupos sociodemográficos, como gênero, possam ser balanceados e um modelo confirmatório ser testado. Sugere-se, ainda, que seja realizada a inversão de alguns pares de adjetivos ao longo da escala, o que pode vir a contribuir para o controle do efeito teto nas respostas. Neste estudo, o afeto foi investigado sob o estímulo das competições para atletas, no entanto o afeto ocorre diariamente e em variados contextos. Assim, sugere-se a investigação da ocorrência do afeto em situações comuns e cotidianas das vidas das pessoas.

Por fim, acredita-se que os resultados produzidos contribuem para o debate nacional e internacional acerca da dimensionalidade do afeto e possíveis formas de mensurá-lo. Espera-se que este estudo contribua para a produção de conhecimento acerca do afeto em território nacional. A escolha de uma perspectiva teórica pouco utilizada no contexto brasileiro teve por objetivo expandir o panorama de conhecimento científico sobre esse construto e, a longo prazo, possibilitar que o afeto possa ser medido de forma mais precisa em diferentes contextos e de variadas formas.

\section{Referências}

Barrett, L. F. (2006). Valence is a basic building block of emotional life. Journal of Research in Personality, 40(1), 35-55. doi: 10.1016/j. jrp.2005.08.006

Betella, A., \& Verschure, P. F. M. J. (2016). The affective slider: A digital self- assessment scale for the measurement of human emotions. PloS One, 11(2), e0148037. doi: 10.1371/journal.pone.0148037 
Bradburn, N. M. (1969). The structure of psychological well-being. Chicago: Aldine Publishing Company.

Byrne, B. M. (2012). Structural equation modeling with mplus basic concepts, applications, and programming. London: London: Routledge Academic. Carroll, J. M., Yik, M. S. M., Russell, J. A., \& Barrett, L. F. (1999). On the psychometric principles of affect. Review of General Psychology, 3(1), 14-22. doi: 10.1037/1089-2680.3.1.14

Carvalho, H. W., Andreoli, S. B., Lara, D. R., Patrick, C. J., Quintana, M. I., Bressan, R. A., Melo, M. F., Mari, J. de J., \& Jorge, M. R. (2013). Structural validity and reliability of the positive and negative affect schedule (PANAS): evidence from a large Brazilian community sample. Revista Brasileira de Psiquiatria, 35(2), 169-172. doi: 10.1590/1516-4446-2012-0957

Crispim, A. A., Roettgers, C., \& Cruz, R. M. (2014). Evidências de validade semântica de um instrumento de medida de afeto. Anais do II Congresso Brasileiro de Psicologia Positiva, Porto Alegre.

Ekkekakis, P. (2013). The measurement of affect, mood, and emotion: A guide for health-behavioral research. Cambridge: Cambridge University Press. Guttman, L. (1954). A new approach to factor analysis: The radex. In P. F. Lazarsfeld (Ed.). Mathematical thinking in the social sciences (pp. 258-348). Glencoe: The Free Press.

Kahneman, D., \& Riis, J. (2005). Living and thinking about it: Two perspectives on life. In A. Huppert, N. Baylis \& B. Keverne (Eds.). The science of well-being (pp. 285-301). Oxford University Press: New York.

Kahneman, D. (2012). Thinking, fast and slow. London: Penguin.

Kammann, R., \& Flett, R. (1983). Affectometer 2: A scale to measure current level of general happiness. Australian Journal of Psychology, 35(2), 259. doi: 10.1007/s11136-006-9145-5

Kline, R. B. (2016). Principles and practice of structural equation modeling (4th ed.) New York: The Guilford Press.

McDonald, R. P. (2014). Test theory: A unified treatment New York: Routledge.

Mehrabian, A., \& Russell, J. A. (1974). An approach to environmental psychology. Cambridge: M. I. T. Press.

Pasquali, L. (2013). Psicometria: Teoria dos testes na psicologia e na educação ( $5^{\text {th }}$ ed.). Petrópolis: Vozes.

Quirin, M., Kazén, M., \& Kuhl, J. (2009). When nonsense sounds happy or helpless: The implicit positive and negative affect test (IPANAT). Journal of Personality and Social Psychology, 97(3), 500-516. doi: 10.1037/a0016063

Russell, J. A. (1980). A circumplex model of affect. Journal of Personality and Social Psychology, 39(6), 1161-1178. doi: 10.1037/h0077714

Russell, J. A. (2003). Core affect and the psychological construction of emotion. Psychological Review, 110(1), 145-172. doi: 10.1037//0033295X.110.1.145

Russell, J. A., \& Barrett, L. F. (1999). Core affect, prototypical emotional episodes, and other things called emotion: dissecting the elephant. Journal of Personality and Social Psychology, 76(5), 805-819. doi: 10.1037/0022-3514.76.5.805

Scherer, K. R., Shuman, V., Fontaine, J. J. R., \& Soriano, C. (2013). The GRID meets the wheel: Assessing emotional feeling via self-report. Oxford University Press. doi: 10.1093/acprof:oso/9780199592746.003.0019

Segabinazi, J. D., Zortea, M., Zanon, C., Bandeira, D. R., Giacomoni, C. H., \& Hutz, C. S. (2012). Escala de afetos positivos e negativos para adolescentes: Adaptação, normatização e evidências de validade. Avaliação Psicológica, 11(1), 1-12.

Seib-Pfeifer, L., Pugnaghi, G., Beauducel, A., \& Leue, A. (2017). On the replication of factor structures of the positive and negative affect schedule (PANAS). Personality and Individual Differences, 107, 201-207. doi: 10.1016/j.paid.2016.11.053

Tabachnick, B. G., \& Fidell, L. S. (2013). Using multivariate statistics (6 ${ }^{\text {th }}$ ed.). Boston: Pearson.

Watson, D., Wiese, D., Vaidya, J., \& Tellegen, A. (1999). The two general activation systems of affect: Structural findings, evolutionary considerations, and psychobiological evidence. Journal of Personality and Social Psychology, 76(5), 820-838. doi: 10.1037/0022-3514.76.5.820

Watson, D., Clark, L. A., \& Tellegen, A. (1988). Development and validation of brief measures of positive and negative affect: The PANAS scales. Journal of Personality and Social Psychology, 54(6), 1063-1070. doi: 10.1037/0022-3514.54.6.1063

Yik, M., Russell, J. A., \& Steiger, J. H. (2011). A 12-point circumplex structure of core affect. Emotion, 11(4), 705-731. doi: 10.1037/a0023980

Yik, M., Russell, J. A., \& Barrett, L. F. (1999). Structure of self- reported current affect: Integration and beyond. Journal of Personality and Social Psychology, 77(3), 600-619. doi: 10.1037/0022-3514.77.3.600

Zanon, C., Bastianello, M. R., Pacico, J. C., \& Hutz, C. S. (2013). Desenvolvimento e validação de uma escala de afetos positivos e negativos. Psico-USF, 18(2), 193-201. doi: 10.1590/S1413-82712013000200003

Zanon, C., \& Hutz, C. S. (2014). Escala de afetos positivos e afetos negativos (PANAS). In C. S. Hutz (Ed.). Avaliação em Psicologia Positiva (pp. 63-68). Porto Alegre: Artmed.

\section{Sobre os autores}

recebido em maio de 2016 reformulado em janeiro de 2017 aprovado em março de 2017

Ana Carla Crispim é doutoranda na Escola de Psicologia da University of Kent, onde desenvolve sua pesquisa sobre a estrutura dimensional do afeto com apoio da CAPES (Processo BEX-2948-13-6).

Roberto Moraes Cruz é professor no Departamento e do Programa de Pós-Graduação em Psicologia da Universidade Federal de Santa Catarina e coordena o Laboratório Fator Humano. Orienta alunos de doutorado e mestrado nas linhas de pesquisa "Processos psicossociais e de saúde no trabalho e nas organizações" e "Avaliação em saúde e desenvolvimento".

Cassandra Melo Oliveira é doutoranda no Departamento de Psicologia da Universidade Federal de Santa Catarina, onde desenvolve sua pesquisa sobre uma bateria adaptativa computadorizada baseada no modelo dos cinco grandes fatores da personalidade e do desenho universal.

Aline Battisti Archer é doutoranda no Departamento de Psicologia e bolsista CAPES-DS da Universidade Federal de Santa Catarina, onde desenvolve sua pesquisa sobre formação profissional. 